\title{
Constitutional Law in Muslim Countries / Challenges for the Afghan Constitution. Impulses from a Comparative Perspective. Doppel-Tagung in Dubai Februar 2009
}

\author{
Von Mina Aryobsei, Heidelberg*
}

Vom 12. bis 16. Februar 2009 trafen sich führende Verfassungsrechtler und Praktiker aus muslimischen Staaten, Europa, Nordamerika, Afrika und Australien im Wirtschaftszentrum der muslimischen Welt in Dubai. Dort veranstaltete das Heidelberger Max-Planck-Institut für ausländisches öffentliches Recht und Völkerrecht (MPI) ein Doppelsymposium zu den Themen „Constitutional Law in Muslim Countries“ sowie „Challenges for the Afghan Constitution - Impulses from a Comparative Perspective“. Der Ort war nicht zufällig gewählt: Nichts lag näher als die Tagung in einem muslimisch geprägten, aber nicht "antiwestlichen" Land durchzuführen. Organisiert hatten die Konferenz die MPI-Mitarbeiter PD Dr. Rainer Grote, Mitherausgeber der OUP-Publikation „Constitutions of the Countries of the World“, und Dr. Tilmann Röder, Leiter der Nahost- und Zentralasienprojekte des Instituts.

In der islamischen Welt vollzieht sich seit dem Ende des vergangenen Jahrhunderts ein fundamentaler Wandel. Die Thematik reflektiert daher eine der wichtigsten rechtlichen Herausforderungen unserer Zeit. Ziel des Symposiums war eine rechtsvergleichende Analyse der Verfassungen muslimischer Staaten. Diskutiert wurden vornehmlich die Rolle der Scharia in den Verfassungen, deren "Hüter" und die Funktion der Gewaltenteilung. Die hierzu vorgetragenen Erkenntnisse sollten der afghanischen Verfassung von 2004 neue Impulse verleihen.

Eröffnet wurde das Symposium durch den Direktor des veranstaltenden Max-PlanckInstituts, Prof. Dr. Dr. h.c. Rüdiger Wolfrum, der zunächst das Institut vorstellte: 1924 als eine unabhängige akademische Institution gegründet, beschäftigt es sich insbesondere mit dem Völker- und Europarecht, dem ausländischen öffentlichen Recht sowie dem deutschen öffentlichen Recht. Das Institut beschränkt sich nicht auf die akademische Seite seiner Themen. So ist es weltweit am Aufbau rechtsstaatlicher Institutionen beteiligt, derzeit vor allem in Afghanistan und im Sudan. Daher war auch eine afghanische Delegation - bestehend aus Mitgliedern der Legislative, der Exekutive und der Judikative - eingeladen, um über aktuelle Schwierigkeiten bei der Implementierung der afghanischen Verfassung zu diskutieren.

Im Mittelpunkt des ersten Symposiumstages stand das Thema „Constitutionalism and the Shari`a: Tensions and Perspectives“. Die unter dem Vorsitz von Prof. Dr. Said Mah-

* Mina Aryobsei, Magistra Legum Europae (M.L.E.).Mitarbeiterin am Institut für Öffentliches Recht der Juristischen Fakultät der Georg-August-Universität Göttingen (Prof. Dr. Christine Langenfeld). E-mail: mina.aryobsei@web.de 
moudi stehende Vormittags-Sitzung begann mit einem Vortrag von Prof. Dr. Hashim Kamali, Direktor des International Institute of Advanced Islamic Studies (Malaysia), zum Thema „Constitutionalism in Muslim Countries: The Perspective of Islamic Law“: Eine Verfassung regele zweierlei: Staatsorganisation und Grundrechte. Das islamische Recht kenne eine solche institutionelle Regelung nicht. Er bezeichnete die islamische Regierungsform als ,civilian“ (madaniyah), sie sei weder theokratisch noch säkular, sondern eine Regierungsform eigener Art. Allerdings sei die Entscheidungsgewalt durch die Regeln des heiligen Koran und der Sunna begrenzt.

In seiner Präsentation zu „Constitutionalism in Muslim Countries: A View from International Law" definierte Rüdiger Wolfrum den Begriff des islamischen Staates mit weiten Kriterien: Islamisch sei ein Staat, in dem die Bevölkerung mehrheitlich dem muslimischen Glauben angehöre oder der sich zu einem islamischen Staat erkläre. Obwohl die islamische Wissenschaft eine Trennung von Staat und Religion nicht akzeptiere, hätten fast alle islamischen Staaten nunmehr eine Verfassung. Dementsprechend habe sich auch das Verhältnis muslimischer Staaten zum Völkerrecht radikal geändert. Zahlreiche Verfassungen islamischer Staaten enthielten Grundrechtskataloge. Der Standard der Menschenrechte im internationalen Recht sei jedoch ein anderer als im Islam. Das unterschiedliche Verständnis der Gleichberechtigung von Frau und Mann bezeichnete er als eine der zwischen internationalem und islamischem Recht meistumstrittenen Positionen. Problematisch seien auch die Vorbehalte muslimischer Staaten gegenüber den internationalen Konventionen. Allerdings seien Erscheinungen wie Vorbehalte und Nicht-Ratifizierung keineswegs typisch für muslimische Staaten. Insgesamt gebe es zwischen internationalem und islamischem Recht sogar weitaus mehr Gemeinsamkeiten, als üblicherweise angenommen.

Dr. Dr. h.c. Adel Omar Sherif, weit über die Grenzen seines Landes bekannter Vizepräsident des ägyptischen Verfassungsgerichts, referierte über das Verhältnis zwischen Verfassung und Scharia in Ägypten. In einem historischen Abriß betonte er die bedeutende Rolle, die der Islam in der ägyptischen Verfassung dank der tiefen Religiosität der Bevölkerung stets gespielt habe. Grundsätzlich schlössen demokratische Staatsform und Islam einander nicht aus. Nach Art. 2 der geltenden ägyptischen Verfassung sollten "die Prinzipien der islamischen Scharia die Hauptquelle der Gesetzgebung" sein. Deswegen sei der Islam aber nicht die ausschließliche Rechtsquelle. Die Scharia sei nur eine Rechtsquelle von mehreren, wenn auch deren bedeutsamste. Folglich stehe die ägyptische Verfassung über der Scharia.

Dr. Zhenis Kembayev, Rechtsprofessor der KIMEP-Universität Almaty (Kasachstan), berichtete über das Verhältnis von Verfassung und Scharia in Kasachstan. Dieses Land sei nach der hier verwendeten Definition ein muslimischer Staat. Der Zusammenbruch der Sowjetunion habe in Kasachstan ungeachtet der ersten unabhängigen Verfassung von 1993 eine politische und wirtschaftliche Krise ausgelöst, bei der eine reformorientierte Exekutive und eine kommunistisch orientierte Legislative sich gegenübergestanden hätten. Unter der reformierten Verfassung von 1995, die sich nicht auf die Scharia oder den Islam beziehe, sei es gelungen, ein effektives Rechtssystem zu entwickeln. Die kasachische Verfassung sei weit entfernt von den Verfassungen beispielsweise Iraks, Irans oder Afghanistans. 
Dr. Kaniye Ebeku von der juristischen Fakultät der Universität Nikosia (Zypern) referierte unter dem Vorsitz von Dr. Asifa Quraishi zum Beispiel Nigeria. Ob Nigeria ein islamischer Staat ist, sei umstritten. Faktisch sei der Süden christlich, der Norden islamisch geprägt. Bis zur britischen Kolonialisierung sei islamisches Recht staatlich anerkanntes Recht gewesen. Mit der britischen Kolonialisierung, bis zu welcher allein islamisches Recht gegolten habe, sei "weltliches" Recht hinzugetreten unter Trennung von der Scharia, ohne dass der im Norden häufig geforderte Vorrang islamischen Rechts sich verfassungsrechtlich durchgesetzt habe. Denn Nigeria, so die Scharia-Gegner, sei ein säkularer Staat. Nigerias Verfassung belege, wie auch ein wenigstens regional muslimisch geprägter Staat Religion und Staat trennen und zudem Menschenrechte und internationale Konventionen beachten könne.

Der französische Conseiller d`Etat und Projektmanager zur Unterstützung des Obersten Gerichtshofs Afghanistans, Thierry Le Roy, referierte über die Verfassungen in den Maghreb-Staaten. Sie unterlägen starken islamischen Einflüssen. Zwar bezögen sie sich alle auf den Islam, die Erforderlichkeit der Übereinstimmung aller Gesetze mit der Scharia werde jedoch nicht verlangt. Islamisches Recht und staatlich gesetztes Recht müssten in einen angemesssenen Ausgleich gebracht werden. Grundsätzlich solle der Gesetzgeber den Einzelnen nicht zu einem Verhalten verpflichten, das im Islam verboten sei; umgekehrt dürften die Pflichten des Islam nicht gesetzlich abgeschafft werden.

Dr. Asem Khalil, Juniorprofessor an der Birzeit University und an der Bethlehem University, berichtete über den Verfassungsprozess in Palästina. Nach der weltweit aktuellen Verfassungsentwicklung sei ein Verfassungstext nicht ausschließlich ein Produkt nationaler Politik. Andere Staaten und internationale Organisationen seien zentrale Akteure der Verfassunggebung. Die Bevölkerung dürfe schließlich über die Annahme oder Ablehnung des jeweiligen Entwurfs entscheiden. Ausländische Verfassungsmodelle ohne Berücksichtigung spezifisch gesellschaftspolitischer Aspekte des jeweiligen Landes stießen erfahrungsgemäß auf Ablehnung. Im Falle der Errichtung eines souveränen palästinensischen Staates werde das palästinensische Grundgesetz durch eine Verfassung ersetzt, die absehbar zur Bildung der nationalen Identität Palästinas beitragen könnte.

Unter dem Vorsitz der afghanischen Juraprofessorin und MPI-Mitarbeiterin Hamida Barmaki begann der zweite, dem Thema der "Institutional Control of Constitutionalism, gewidmete Tag des Symposiums. Zunächst stellte PD Dr. Rainer Grote (MPI) eine rechtsvergleichende Analyse zu diversen Modellen der institutionellen Verfassungskontrolle vor. $\mathrm{Zu}$ unterscheiden seien drei verschiedene Modelle: die politische, theokratische und richterliche Kontrolle. Die meisten nord- und westafrikanischen Staaten sowie der Mittlere Osten würden dem französischen Model der abstrakten Normenkontrolle folgen. Dieses sei zentralistisch angelegt. Problematisch daran sei, dass Minderheitenrechte und Rechte des Einzelnen nicht hinreichend beachtet würden. Länder, die dem Einfluss des Common Law unterliegen, hätten hingegen ein dezentrales System zur Verfassungskontrolle entwickelt. Nach diesem Modell hätten zwar alle Gerichte die Befugnis, sich mit Verfassungsfragen zu beschäftigen, die letzte Entscheidung stehe jedoch dem Obersten Gerichtshof zu. Aufgrund 
der häufig auftretenden zentralistischen Staatsstruktur spiele die Verfassungsgerichtsbarkeit entweder keine große Rolle oder sie werde in einigen Ländern politisiert. So hätte in der Türkei das Verfassungsgericht Dutzende politischer Parteien verboten, die angeblich die säkulare oder nationale Basisstruktur des Staates bedroht hätten. Im Iran habe der Wächterrat eine Schlüsselrolle in der Eliminierung reformorientierter Kandidaten gespielt. Zudem würden Verfassungsgerichte in islamischen Staaten oft unter politisch schweren und unstabilen Verhältnissen arbeiten. Diese Probleme hätten erfahrene Demokratien nicht.

Dr. Imen Gallala vom Max-Planck-Institut für ausländisches und internationales Privatrecht in Hamburg stellte die Verfassungsgerichtsbarkeit Tunesiens vor. Die politische Elite Tunesiens halte bereits eine Verfassung für ausreichend, um Machtmissbrauch zu verhindern. Die Verfassungen von 1959 - 1987 hätten keine Regelung zur institutionellen Kontrolle gekannt. Nach dem Regierungswechsel am 7. November 1987 hätte der neue Präsident Ben Ali versprochen, die staatlichen Institutionen sowie das Rechtsstaatsprinzip zu stärken. Im Zuge dessen habe er den Verfassungsrat gegründet. Dieser habe nur auf Initiative des Präsidenten die Verfassungsmäßigkeit der Gesetze überprüfen können. Inzwischen habe er eine verfassungsrechtlich gefestigte Stellung. Auch wenn es eine Individualbeschwerde nach wie vor nicht gebe, sei die Verfassungsgerichtsbarkeit in Tunesien auf einem guten Weg.

Dr. Hamid Khan, Anwalt am Obersten Gerichtshof von Pakistan und ausgewiesener Verfassungsrechtler, berichtete über die institutionelle Verfassungskontrolle in Pakistan. Wie die meisten Common-Law-Länder folgt Pakistan dem Supreme Court-Modell. Eine Entscheidung des Obersten Gerichtshofs sei nach dem Prinzip der stare decicis bindend für alle anderen Gerichte. In seiner sechzigjährigen Geschichte habe das Land viele Krisen erfahren. Militärdiktaturen hätten überwiegend die Politik Pakistans bestimmt. In Folge dessen sei das Kriegsrecht ausgerufen worden. Der pakistanischen Justiz wäre es kaum möglich gewesen, frei und unabhängig zu arbeiten. Durch ihre Arbeit hätte sie nicht die Verfassung oder ihre Institutionen geschützt, sondern vielmehr zur Legitimierung der Militärregime beigetragen. Die Gerichtsbarkeit hätte sich jedoch immer dann bewiesen, wenn ihr die Möglichkeit gegeben wurde, unabhängig zu arbeiten. Vor allem unter Iftikhar Muhammad Chaudhry, dem ehemaligen Präsidenten des Obersten Gerichtshofs, hätte sich das Gericht - zum Ärger von General Musharraf - bemüht, zum Wohle der Bevölkerung zu arbeiten. Daraufhin habe ihn General Musharraf entlassen und mit seiner Familie unter Hausarrest gestellt. Es sei daher sehr zufriedenstellend, dass gerade der Streit um die Unabhängigkeit der Justiz Parvez Musharraf zum Rücktritt gezwungen hätte.

Prof. Dr. Hoong Phun Lee, Vizedekan der Juristischen Fakultät der für SüdostasienStudien bekannten Monash University (Australien), referierte über die institutionelle Kontrolle der malaysischen Verfassung. Anders als die Verfassungen vieler muslimischer Staaten, die in Folge militärischer Auseinandersetzung stets einer Änderung bzw. Erneuerung unterzogen worden seien, hätte die malaysische Verfassung im Jahre 2007 ihren 50. Jahrestag gefeiert. Der Islam sei zwar nach Art. 3 der malaysischen Verfassung Staatsreligion, jedoch gelte der Vorrang der Verfassung nach Art. 4. Hüter der Verfassung sei die Ge- 
richtsbarkeit, wobei an höchster Stelle das Bundesgericht (Federal Court) stehe. So hätte das Gericht beispielsweise in dem Fall Public Prosecutor v Dato `Yap Peng ${ }^{l}$ ein Bundesgesetz, welches die Gerichtsbarkeit des Bundes verletzt hätte, angefochten. Am Beispiel Malaysias werde deutlich, dass auch ein Entwicklungsland eine unabhängige Instanz zur Kontrolle der Verfassung haben könne. Auch für die afghanische Verfassung sei eine unabhängige Justiz unabdinglich. Zudem müsste die Verfassung für die Regierungsträger selbst ein unabänderliches Dokument darstellen.

Am Nachmittag des zweiten Tags des Symposiums wurden die Voträge unter dem Vorsitz von Dr. Abdul Wahab Abdul (VAE) fortgeführt. Mit Prof. Dr. Seyed Mohammad Hashemi, Professor für öffentliches Recht an der Juristischen Fakultät der Shahid-Behéshti Universität (Iran), sprach ein führender schiitischer Rechtsgelehrter über die institutionelle Kontrolle der iranischen Verfassung. Hashemi wies darauf hin, dass in Iran der Vorrang der Verfassung gelte. Die Verfassung Irans basiere auf der Achtung der Menschenrechte und Demokratie. Zudem proklamiere sie die Gleichberechtigung aller Menschen. Art. 9 untersage es Menschenrechte zu verletzen, Art. 4 verlange, dass alle Gesetze in Einklang mit den islamischen Maßstäben stehen. Er stellte fest, dass die iranische Bevölkerung der Ansicht sei, die Scharia sei mit der Demokratie kompatibel.

Feisal Amin Rasoul Istrabadi, stellvertretender ständiger Vertreter des Iraks in den Vereinten Nationen und Mitverfasser der Interimverfassung aus den Jahren 2004-2005, referierte über den „Menschenrechtsschutz im Irak“. Der Irak sei bisher unter acht Verfassungen regiert worden. Die Normen der aktuellen Verfassung von 2006 zeigten, dass im Irak - auch wenn nur theoretisch - eine Rechtstradition gilt. Der Islam werde als ,principal source of legislation“" gesehen. Art. 2 der Verfassung beinhalte unter anderem die Klausel, dass kein Gesetz gegen die Bestimmungen des Islams verstoßen dürfe. Diese Norm könne auch dahingehend interpretiert werden, dass sie eine verfassungsrechtliche Inkorporierung der Scharia proklamiere. Gleichzeitig verlange Art. 2 der Verfassung, dass kein Gesetz „may be enacted which contradicts the fundamental rights and freedoms contained in this Constitution“. Angesichts dieses Absatzes sei jedoch nicht ganz klar, welche Rechtsquelle in der Hierarchie höher stünde. Zudem sei nicht geregelt, welche Institution auszulegen hätte, was genau unter ,the fixed principles of Islam“ zu verstehen sei. Hinsichtlich der Gewährleistung von Menschenrechten und der Rechte von Angeklagten sei überraschend zu beobachten, dass diese Rechte zumindest auf dem Papier geschützt worden seien. Der Referent schloß daraus, gerade weil es im Irak eine Rechtskultur gebe, bestehe Hoffnung für die Zukunft des Landes.

Am dritten Tag wurde das Symposium unter Vorsitz von Dr. Kilian Bälz mit der Thematik der „Separation of Powers“ fortgesetzt. Im Einführungsvortrag stellte Dr. Tilmann Röder (MPI), eine rechtsvergleichende Perspektive hinsichtlich der Gewaltenteilung und ihrer Wurzeln vor. Diese gehe von einer Trennung der Gewalt zwischen verschiedenen Institutionen aus. Montesquieus horizontale Gewaltenteilung richte sich gegen Macht- 
mißbrauch. Es werde allgemein vertreten, dass die Idee der Gewaltenteilung erst nach dem ersten Weltkrieg in der muslimischen Welt Geltung erhalten habe. Zu erforschen sei jedoch, ob sie bereits Ende des 19. und Anfang des 20. Jahrhunderts in der osmanischen Verfassung Eingang fand. Dies sei in der Forschung noch stark umstritten. In der islamischen Welt finde man heute verschiedene Verfassungsmodelle vor. Im Hinblick auf die Gewaltenteilung müsse zwischen den Verfassungen mehr oder weniger demokratischer Staaten und denjenigen von Monarchien und autoritären Regimen unterschieden werden. Demokratische Staaten würden - zumindest auf dem Papier - eine Teilung der Gewalten vorschreiben, während letztere - wenn überhaupt - das Gebot der Gewaltenteilung nur fragmentarisch beachten würden.

Dr. Martin Lau, Dozent an der School of Oriental and African Studies (SOAS) of the University of London, referierte über die gerichtliche Nachprüfung in Pakistan. Darunter seien zwei verschiedene Konzepte zu verstehen. Zum einen die gerichtliche Kontrolle von Exekutivhandlungen, wie sie Art. 4 der pakistanischen Verfassung vorsehe, wonach jedermann gesetzeskonform zu behandeln sei. Dagegen sehe der ,judicial review“ die Befugnis einiger Gerichte zur Überprüfung der Verfassungsmäßigkeit von Gesetzen vor. Mit der Errichtung des 1980 gegründeten Federal Shariat Court (FSC) habe die gerichtliche Überprüfung eine neue Richtung angenommen. Das Gericht könne Gesetze auf ihre Vereinbarkeit mit dem islamischem Recht überprüfen. Wegen Verstoßes gegen islamisches Recht könne das Gericht das Gesetz für ungültig erklären. Hauptkritikpunkt sei seine Entscheidungsbefugnis darüber, was islamisches Recht sei. Dass dies vor 1980 Aufgabe der Regierung gewesen sei, werde vielfach als demokratischer und gerechter erachtet.

Im darauf folgenden Vortrag trug Dr. Cordelia Koch ihre Forschungsergebnisse über die Gewaltenteilung im Libanon vor. Die Mehrheit der Bevölkerung gehöre zwar zum muslimischen Glauben, der Staat sei aber als christlicher Staat gegründet worden. Eine Gewaltenteilung im Libanon existiere faktisch nicht. Die Missfunktion der Gerichte sei seit dem libanesischen Bürgerkrieg (1975-1990) nunmehr Normalzustand. Libanons Hauptproblem sei jedoch das Nichtfunktionieren der Legislative zum einen und der Exekutive zum anderen. Diese Tatsachen seien nicht allein auf den Bürgerkrieg zurückzuführen, da das Verhältnis zwischen diesen Gewalten schon immer problematisch gewesen sei. Dabei sei zwischen der Verfassung vor und nach dem Krieg zu differenzieren. Die Vor-Kriegsverfassung habe dem Präsidenten eine zentrale Rolle zugeteilt. Er sei weder dem Parlament Rechenschaft schuldig gewesen noch hätte es nach seiner Wahl eine Möglichkeit gegeben, ihn wieder abzusetzen. Die weitgehenden Befugnisse des maronitischen Präsidenten seien Hauptkritikpunkt der Vorkriegs-Verfassung gewesen. Um den Bürgerkrieg zu Ende zu bringen, sei die Verfassung 1990 schließlich geändert worden. Nunmehr seien die drei höchsten Staatsämter bestimmten religiösen Gruppen vorbehalten. So müsse der Staatsoberhaupt maronitischer Christ, der Regierungschef sunnitischer Muslim und der Parlamentspräsident schiitischer Muslim sein. Zudem sei nach Art. 64 der Regierungschef dem Staatsoberhaupt gleichgestellt, indem jede Entscheidung einer Gegenzeichnung des anderen Amtsträgers bedürfe. Ferner müsse nach Art. 65 innerhalb der Regierung nach dem 
Konsensprinzip entschieden werden. Statt die Macht im Sinne Montesquieus strikt zu teilen, verteile die libanesische Verfassung sie nur.

„Substantive Constitutional Law in Afghanistan“ war das Thema des letzten Tags des Symposiums. Den Vorsitz hatte am Vormittag der Anwalt und Friedensmediator aus Bangladesh, Dr. Kamal Hossain. Als erster Referent des Tages hielt Dr. Mohammad Qasim Hashimzai, stellvertretender Justizminister Afghanistans, einen Vortrag über die Gewaltenteilung in der afghanischen Verfassung. Der Referent betonte, es sei wichtig, dass eine Institution über die Einhaltung der Verfassung wache. In Betracht käme der Oberste Gerichtshof. Es sei umstritten, ob dieser mit Blick auf Art. 121 auch die Befugnis zur Auslegung der Verfassung besitze. ${ }^{2}$ Daher müsse in der Verfassung explizit geregelt werden, welche Institution zu ihrer Interpretation und Auslegung befugt sei. Unklar seien zum Beispiel die Art. 83 Abs. 2 und 160 Abs. 3 der Verfassung. Art. 83 Abs. 2 der afghanischen Verfassung regele das Datum der Wahl zur Volksvertretung, die alle fünf Jahre stattfinden müsse. Nach Art. 160 Abs. 3 werden alle Anstrengungen unternommen damit die erste Präsidentschaftsnorm und die erste Wahl zur Nationalversammlung gleichzeitig stattfänden. Aufgrund der Tatsache, dass in der der Präsident seine Arbeit letzten Periode mit einem Verzug von sechs Monaten aufgenommen hatte, sei nun umstritten wie mit dieser Vorschrift zu verfahren sei. Die Regierung behaupte, dass der Präsident fünf Jahre im Amt bleiben müsse. Dieses Problem bedürfe einer Klärung durch den Obersten Gerichtshof. Abschließend stellte er klar, dass eine mögliche Verfassungsänderung nur in Kooperation mit der Loya Jirga durchzuführen sei.

J. Alexander Thier, Rechtsberater am Friedensinstitut der Vereinigten Staaten, referierte über „eine Krise für die afghanische Verfassung?“. Er verwies weitestgehend auf den Vortrag von Dr. Mohammad Qasim Hashimzai. Es müsse geklärt werden, welche Institution die Befugnis zur Auslegung der Verfassung habe. Hierzu verwies er auf den Fall des Außenministers Spanta. Als das Parlament gegen diesen einen Misstrauensantrag gestellt hatte, habe die Regierung die Befugnis des Parlaments hierzu nicht anerkannt und den Obersten Gerichtshof angerufen. Die Entscheidung des Obersten Gerichtshofs sei wiederum vom Parlament nicht anerkannt worden. Zu der Problematik der Interpretation der Verfassung fügte er noch Folgendes hinzu: Nach Art. 3 der Verfassung dürfe in Afghanistan kein Gesetz den Bestimmungen des Islams widersprechen. In Verbindung mit Art. 121 könne dies so verstanden werden, dass die Überprüfung dieser Frage dem Obersten Gerichtshof obliegt. Dasselbe könne man jedoch auch im Hinblick auf Art. 157 annehmen, der vorschreibt, dass eine „Unabhängige Kommission zur Überwachung der Einhaltung der Verfassung" gebildet werde.

Art. 121 Afg.Verf.: Zu den Befugnissen des Obersten Gerichtshofs gehören auf Ersuchen der Regierung oder der Gerichte die Überprüfung der Gesetze, Gesetzeserlasse, internationalen Verträge und Konventionen auf Übereinstimmung mit der Verfassung und deren Auslegung nach Maßgabe der gesetzlichen Bestimmungen. 
Mit dem Vortrag von Richter Bahauddin Baha, stellvertretender Präsident des Obersten Gerichtshof Afghanistans, zur „Befugnis des Obersten Gerichtshofs zur Rechtsprechung in Verfassungsangelegenheiten“, setzte sich das Symposium fort. Bahauddin Baha konzentrierte sich weitgehend auf die Entstehung der Gerichtsbarkeit in Afghanistan. Diese habe sich in den letzten Jahrhunderten gewaltig verändert. Die Prinzipien der Gerichtsbarkeit seien in Afghanistan zum ersten Mal in der Verfassung von 1923 kodifiziert worden. Der Oberste Gerichtshof sei durch die Verfassung von 1964 als die höchste richterliche Instanz gegründet worden. Die neue afghanische Verfassung von 2004 beinhalte diverse Artikel zur Organisation und dem System der Gerichtsbarkeit. Der Oberste Gerichtshof sei eine unabhängige Institution zur Überprüfung der Rechtsprechung und dem Handeln der unteren Gerichtsinstanzen. Um dies zu erreichen böte der Gerichtshof den unteren Instanzen Hilfe zur Lösung derer Probleme an. Gerechtigkeit sei das einzige Ziel des Obersten Gerichtshofes.

Die Parlamentarierin Shukria Barakzai, LL.M., führte in ihrem Referat über das Konzept der „Kommission zur Überprüfung der Umsetzung der afghanischen Verfassung“ nach Art. 157 sehr anschaulich die Abgrenzung der Befugnisse der Kommission und des Obersten Gerichthofs vor Augen. Der Kommission komme eine Aufsichtsrolle zu. Dies sei nicht mit der Auslegung der Verfassung, die dem Obersten Gerichtshof zustehe, gleichzusetzen. Nach Art. 157 Abs. 2 Afg.Verf werden die Mitglieder der Kommission vom Staatspräsidenten mit Zustimmung der Volksvertretung ernannt. Damit sei ihre Unabhängigkeit nicht garantiert. Daher sei die Verfassungskontrolle dem Obersten Gerichtshof als unabhängige Instanz zu überlassen. Diese Verantwortung könne die Kommission nicht übernehmen. Eine Verfassungsänderung lehne sie derzeitig ab. Grundsätzlich müsse die afghanische Verfassung mehr Beachtung finden. Der Grundsatz der Rechtsstaatlichkeit müsse sich durchsetzen. Dazu bedürfe es einer starken Regierung, die den Schutz ihrer Bürger in den Vordergrund stellt.

Prof. Dr. Mohammad Hashim Kamali fuhr mit der Kommentierung der Art. 121 und 157 der afghanischen Verfassung fort. Für ihn bestehe ein generelles Problem der Umsetzung der Verfassung. Das Land kenne keine gefestigte Verfassungskultur. Der Präsident sei zum Hüter der Verfassung berufen. In dem Spanta-Fall habe sich dieser jedoch auf die Seite des Ministers gestellt. Er müsste sich in erster Linie für die Umsetzung der Verfassung einsetzen. Im Hinblick auf Art. 121 habe allein der Oberste Gerichtshof als unabhängige Instanz die Befugnis zur Auslegung der Verfassung. Im Rahmen der Verfassunggebung habe die Loya Jirga jedoch befürchtet, auch in Afghanistan könnte eine, dem iranischen Wächterrat ähnliche, Institution entstehen. Daher sei diese Aufgabe dem Obersten Gerichtshof als Judikativorgan übertragen worden. Sicherlich beinhalte der Text des Art. 121 inhaltliche Schwächen. Es müsse auch geklärt werden auf wessen Initiative der Oberste Gerichtshof handeln kann. Daher bedürfe der Begriff der „Regierung“ einer genauen Bestimmung. Z.B. sei unklar, ob auch das Parlament unter diese Begrifflichkeit zu subsumieren sei. Die Kommission sei ein Exekutivorgan. Aufgrund der Gewaltenteilung dürften dieser daher nicht Aufgaben der Judikative übertragen werden. 
Anschließend wurde eine offene Diskussion über die Auslegung der Verfassung geführt. Prof. Dr. Dr. h. c. Rüdiger Wolfrum differenzierte im Hinblick auf Art. 121 zwischen einer Überprüfung der Verfassungsmäßigkeit und der Verfassungsauslegung an sich. Die Verfassungsauslegung sei Aufgabe jeder Institution, die die Verfassung umsetze. Die Überprüfungskompetenz sei jedoch begrenzt und solle von der Regierung und allen Gerichten angewandt werden. Es sei wichtig allein auf den Text der Verfassung zu schauen. Ein historischer Blick könne schaden.

Fazel Ahmad Manawi, Mitglied der afghanischen Wahlkommission, äußerte sich zu Art. 121 der Verfassung. Lange habe der Oberste Gerichtshof die Verfassung nach Maßgabe des Art. 121 interpretiert. Das Problem sei jedoch, dass im Namen der Verfassungsauslegung vielmehr eine Änderung der Verfassung stattfände und somit die jeweiligen Vorschriften missbraucht würden. Diese Aufgabe solle daher eine unabhängige Instanz, die das Vertrauen der Bevölkerung gewinnen könne, übernehmen.

Unter dem Vorsitz des Institutsdirektors Wolfrum setzte sich das Symposium am letzten Halbtag fort. Prof. Dr. Ali Wardak, Dozent für Kriminologie an der Universität Glamorgan (GB), hielt einen Vortrag über die „Beziehung der Scharia und der afghanischen Verfassung“. Die Scharia sei ein wichtiger Bestandteil für die Rechtsfindung in Afghanistan. Zudem existiere in Afghanistan das Stammesrecht. $80 \%$ der Streitfälle würden von den Schuras und Dschirgas genannten Stammesräten gelöst werden. Diese seien weitaus weniger korrupt als die staatlichen Gerichte. Problematisch sei jedoch, dass diese ausschließlich von Männern besetzt seien. Die Sachlichkeit sei vor allem dann nicht gewahrt, wenn diese in einem Streitfall zwischen den Geschlechtern entscheiden müssten. Das Land brauche daher ein formales Rechtssystem, das transparent sei. Zwar habe Afghanistan diverse internationale Verträge unterschrieben, ihre Umsetzung müsste jedoch besser mit der internationalen Gemeinschaft abgesprochen werden. Die Normenhierarchie sei wie folgt von der Verfassung vorgegeben: An höchster Stelle stehe die Verfassung. Diese werde vom staatlich gesetzten Recht gefolgt. Die Scharia sei an dritter Stelle zu setzen. Zum Schluss folge das Stammesrecht, das nicht in der Verfassung erwähnt sei. Lediglich das afghanische Zivilgesetzbuch beziehe sich in Art. 2 subsidiär auf das Stammesrecht.

Abschließend wurde eine Podiumsdiskussion unter den Referenten des Tages geführt. Nach Dr. Mohammad Qasim Hashimzais Ansicht sei die Verfassung hinsichtlich der Normenhierarchie klar: Kein Gesetz dürfe den Bestimmungen des Islam widersprechen. Das Strafrecht und das Bürgerliche Recht seien faktisch kodifiziertes islamisches Recht. In erster Linie stehe die Verfassung, und alle anderen Gesetze seien ohnehin islamisches Recht. Eine Verfassungsänderung lehnte er ab. Prof. Mohammad Hashim Kamali fügte hinzu, dass zwar Fortschritte erkennbar seien, die Gesamtsituation jedoch frustrierend. Auch er sei gegen eine Verfassungsänderung. Das sei eine unnötige Verschwendung von Ressourcen. Man müsse sich vielmehr auf die Umsetzung der Verfassung konzentrieren. Beispielhaft stellte er dar, ein schlechter Richter könne auch keine vertretbare Entscheidung mit einem ,guten“ Gesetz treffen. Hingegen würde ein ,guter“ Richter auch ein schlechtes Gesetz auf angemessene Weise anwenden können. Das Land brauche nicht die beste Ver- 
fassung der Welt. Die Priorität müsse zunächst bei den Problemen der Bevölkerung liegen. Er hoffe auf den Einsatz der internationalen Gemeinschaft für die afghanische Bevölkerung. Derzeit fehle die Basis für jegliches Handeln. Kriegsverbrecher sollten vor den internationalen Strafgerichtshof gebracht werden. Die „Gerechtigkeit“ in Afghanistan müsse wiederhergestellt werden. Die Korruptionsrate sei so hoch wie noch nie zuvor. In erster Linie sollte diese bekämpft werden. Afghanistan brauche eine effektive Regierung. Zudem seien ein starkes Militär und eine effektiv arbeitende Polizei erforderlich. Präsident Karsai habe derzeit nicht den nötigen Einfluss, um Warlords und Drogenbarone zu entmachten. Um Rechtsstaatlichkeit durchzusetzen, sei eine effektive Gerichtsbarkeit essentiell. Derzeit würden einigen Gerichten nicht einmal die geltenden Gesetzestexte zur Verfügung stehen. Im Rahmen des Wiederaufbaus habe man der Gerichtsbarkeit nicht genügend Aufmerksamkeit geschenkt. Das müsse sich ändern.

Prof. Dr. Dr. h.c. Rüdiger Wolfrum brachte die Diskussion zu einem Abschluss. Er habe aus der Debatte zwei Botschaften entnehmen können. Zum einen solle man sich auf die aktuelle Verfassung und die gegebenen rechtlichen Rahmenbedingungen konzentrieren. Afghanistan bräuchte keine neue Regeln und Gesetze. Zum anderen solle man stets optimistisch sein. Nun hoffe er, dass sich das auch bewähren werde. ${ }^{3}$ val and Continuity“ bei Oxford University Press im Sommer 2010 erscheinen. 